\title{
AN EXTENSION OF ITO'S DIFFERENTIATION FORMULA
}

\author{
ATA N. AL-HUSSAINI AND ROBERT J. ELLIOTT
}

Introduction 1. If $L_{t}^{a}$ denotes the local time of a continuous semimartingale $X$ at a Bouleau and Yor [1] have obtained a form of Ito's differentiation formula which states that for absolutely continuous functions $F(x)$

$$
F\left(X_{t}\right)=F\left(X_{0}\right)+\int_{0}^{t} \frac{\partial F}{\partial x}\left(X_{s}\right) d X_{s}-\frac{1}{2} \int_{-\infty}^{\infty} \frac{\partial F}{\partial x}(a) d_{a} L_{t}^{a} .
$$

In [5] Yor uses this expression to discuss the approximations obtained by Yamada [4] to 'zero energy' processes. This article extends these ideas to suitable functions of the form $F(t, x)$. In fact, for a continuous semimartingale $X_{t}, t \geq 0$, with local time $L_{t}^{a}$ at $a$, (which may be taken to be jointly right continuous in $a$ and $t$, left limited in $a$ and continuous in $t$ ), and a function $F$ which is $C^{1}$ in $t$, and for which $F(t, x)$ and $(\partial F / \partial t)(t, x)$ are absolutely continuous in $x$, with bounded derivatives, the following differentiation formula holds:

$$
\begin{aligned}
F\left(t, X_{t}\right)= & F\left(0, X_{0}\right)+\int_{0}^{t} \frac{\partial F}{\partial t}\left(s, X_{s}\right) d s+\int_{0}^{t} \frac{\partial F}{\partial x}\left(s, X_{s}\right) d X_{s} \\
& -\frac{1}{2} \int_{-\infty}^{\infty} \frac{\partial F}{\partial x}(t, a) d_{a} L_{t}^{a}+\frac{1}{2} \int_{0}^{t} \int_{-\infty}^{\infty} \frac{\partial^{2} F}{\partial t \partial x}(s, a) d_{a} L_{s}^{a} d s .
\end{aligned}
$$

An advantage of this expression is that only differentiability to the first order in $x$ is required.

Assumptions 2. In the sequel $X$ will denote a real, continuous semimartingale $\left\{X_{t}, t \geq 0\right\}$ defined on a filtered probability space $(\Omega, F, \underline{F}, P)$ which satisfies the usual conditions. Write $T_{n}=\inf \left(t:\left|X_{t}\right| \geq n\right)$. By localizing, that is by considering $X^{T_{n}}$, we can suppose that $X$ is bounded. We shall take the version of the local time $L_{t}^{a}$ with the above continuity properties in $a$ and $t$.

Received May 13, 1985. 
Remarks 3. A key step in formulae (1) and (2) is the definition of the integrals with respect to $d_{a} L_{t}^{a}$ for fixed $t \geq 0$. Recall Tanaka's formula for the local time at $a$ :

$$
\left(X_{t}-a\right)^{-}=\left(X_{0}-a\right)^{-}-\int_{0}^{t} I_{X_{s} \leq a} d X_{s}+\frac{1}{2} L_{t}^{a} .
$$

By initially considering step functions of the form

$$
f(u)=\sum_{i=1}^{n} f_{i} I_{] a_{i}, a_{i+1}\right]}(u),
$$

and linear combinations of expression (3), Bouleau and Yor [1] show that if $F(x)=\int_{0}^{x} f(u) d u$ then

$$
F\left(X_{t}\right)=F\left(X_{0}\right)+\int_{0}^{t} f\left(X_{s}\right) d X_{s}-\frac{1}{2} \int_{-\infty}^{\infty} f(a) d_{a} L_{t}^{a},
$$

where the last integral is the sum

$$
\sum_{i=1}^{n} f_{i}\left(L_{t}^{a_{i+1}}-L_{t}^{a_{i}}\right)
$$

It is shown this map can be extended to a vector measure on the Borel field of $R$ with values in $L^{2}(\underline{F}, P)$, so that if $f: R \rightarrow R$ is a locally bounded Borel measurable function and $F(x)=\int_{0}^{x} f(u) d u$ then $F\left(X_{t}\right)$ is given by (4). Indeed, if $F(x)$ is any absolutely continuous function with a locally bounded derivative then $F\left(X_{t}\right)$ is given by (4), because, writing $G(x)=F(x)-F(0)=\int_{0}^{t}(\partial F / \partial x)(u) d u$, the result is valid for $G\left(X_{t}\right)$.

Lemma 4. Suppose $f: R \rightarrow R$ is $C^{1}$. Then for any $t$ :

$$
\begin{aligned}
\int_{-\infty}^{\infty} f(a) d_{a} L_{t}^{a} & =-\int_{0}^{t} \frac{\partial f}{\partial x}\left(X_{s}\right) d\langle X, X\rangle_{s} \\
& =-\int_{-\infty}^{\infty} \frac{\partial f}{\partial x}(a) L_{t}^{a} d a
\end{aligned}
$$

Proof. Write $F(x)=\int_{0}^{x} f(u) d u$. Then applying the Ito differentiation formula to $F\left(X_{t}\right)$ :

$$
F\left(X_{t}\right)=F\left(X_{0}\right)+\int_{0}^{t} f\left(X_{s}\right) d X_{s}+\frac{1}{2} \int_{0}^{t} \frac{\partial f}{\partial x}\left(X_{s}\right) d\langle X, X\rangle_{s} .
$$


Equating the final terms of (4) and (5) the result follows. However, we also have from [2], p. 368, that

$$
\int_{0}^{t} \frac{\partial f}{\partial x}\left(X_{s}\right) d\langle X, X\rangle_{s}=\int_{-\infty}^{\infty} \frac{\partial f}{\partial x}(a) L_{t}^{a} d a
$$

Remark 5. For absolutely continuous $f$

$$
\int_{-\infty}^{\infty} f(a) d_{a} L_{t}^{a}=-\int_{-\infty}^{\infty} \frac{\partial f}{\partial x}(a) L_{t}^{a} d a
$$

and treating the $t$ in the function as a constant, we also have for functions $f(t, x)$ which are absolutely continuous in $x$,

$$
\int_{-\infty}^{\infty} f(t, a) d_{a} L_{t}^{a}=-\int_{-\infty}^{\infty} \frac{\partial f}{\partial x}(t, a) L_{t}^{a} d a .
$$

The generalized differentiation formula is first established for a suitably smooth function $f(t, x)$.

Theorem 6. Suppose, for $(t, x) \in[0, \infty) \times R, F(t, x) \in R$ is continuously differentiable in $t$ and twice continuously differentiable in $x$. Then

$$
\begin{aligned}
F\left(t, X_{t}\right)= & F\left(0, X_{0}\right)+\int_{0}^{t} \frac{\partial F}{\partial t}\left(s, X_{s}\right) d s+\int_{0}^{t} \frac{\partial F}{\partial x}\left(s, X_{s}\right) d X_{s} \\
& -\frac{1}{2} \int_{-\infty}^{\infty} \frac{\partial F}{\partial x}(t, a) d_{a} L_{t}^{a}+\frac{1}{2} \int_{0}^{t} \int_{-\infty}^{\infty} \frac{\partial^{2} F}{\partial t \partial x}(s, a) d_{a} L_{s}^{a} d s .
\end{aligned}
$$

Proof. By Ito's differentiation formula:

$$
\begin{aligned}
F\left(t, X_{t}\right)= & F\left(0, X_{0}\right)+\int_{0}^{t} \frac{\partial F}{\partial t}\left(s, X_{s}\right) d s+\int_{0}^{t} \frac{\partial F}{\partial x}\left(s, X_{s}\right) d X_{s} \\
& +\frac{1}{2} \int_{0}^{t} \frac{\partial^{2} F}{\partial x^{2}}\left(s, X_{s}\right) d\langle X, X\rangle_{s} .
\end{aligned}
$$

Recall we are taking $X=X^{T_{n}}$ so $\left(\partial^{2} F / \partial x^{2}\right)\left(s, X_{s}\right)$ is continuous and bounded for $s \leq t$. Again from [2], p. 368,

$$
\int_{0}^{t} \frac{\partial^{2} F}{\partial x^{2}}\left(s, X_{s}\right) d\langle X, X\rangle_{s}=\int_{-\infty}^{\infty} \int_{0}^{t} \frac{\hat{o}^{2} F}{\partial x^{2}}(s, a) d_{s} L_{s}^{a} d a
$$

Integrating the inner integral by parts in $s$ this is

$$
=\int_{-\infty}^{\infty}\left(L_{t}^{a} \frac{\hat{o}^{2} F}{\partial x^{2}}(t, a)-\int_{0}^{t} L_{s}^{a} \frac{\partial^{3} F}{\partial t \partial x^{2}}(s, a) d s\right) d a
$$

Using Fubini's Theorem to interchange the order of integration, ( $L^{a}$ has 
compact support), and then integrating by parts in $a$ this equals:

$$
-\int_{-\infty}^{\infty} \frac{\partial F}{\partial x}(t, a) d_{a} L_{t}^{a}+\int_{0}^{t} \int_{-\infty}^{\infty} \frac{\partial^{2} F}{\partial t \partial x}(s, a) d_{a} L_{s}^{a} d s .
$$

Substituting in (7) the result follows.

Remarks 7. When $X$ is Brownian motion Perkins, [3], has shown that $L_{t}^{a}$ is a semimartingale in $a$ for each $t \in[0, \infty)$. Yor, [5], has pointed out, using the monotone class theorem, that the integral with respect to $d_{a} L_{t}^{a}$ then equals the stochastic integral in $a$. The advantage of the differentiation formula in the form given by Theorem 6 is that, as stated, it requires only differentiability of order one in $x$. Following the usual mollifier techniques we show that the result holds under a weaker differentiability hypothesis.

Corollary 8. Suppose that $F(t, x)$ is continuously differentiable in $t$ and absolutely continuous in $x$ with a locally bounded derivative $\partial F / \partial x$. Furthermore, suppose that $F(t, 0)=0$ so that for all $t \geq 0$

$$
F(t, x)=\int_{0}^{x} \frac{\partial F}{\partial x}(t, y) d y .
$$

Similarly, suppose that for all $t \geq 0$

$$
\frac{\partial F}{\partial t}(t, x)=\int_{0}^{x} \frac{\partial^{2} F}{\partial t \partial x}(t, y) d y
$$

where $\partial^{2} F / \partial t \partial x$ is locally bounded. Then $F\left(t, x_{t}\right)$ is given by the differentiation formula (6) of Theorem 6.

Proof. Write $f(t, y)=(\partial F / \partial x)(t, y)$. Suppose $g \in C_{0}^{\infty}(R)$ is such that $\int g(x) d x=1$, and for each integer $n>0$ put

$$
\begin{aligned}
F_{n}(t, x) & =n \int F(t, x-y) g(n y) d y \\
& =n \int F(t, y) g(n(x-y)) d y .
\end{aligned}
$$

Then

$$
\frac{\partial F_{n}}{\partial x}(t, x)=n \int f(t, x-y) g(n y) d y,
$$

and 


$$
\frac{\partial^{2} F_{n}}{\partial t \partial x}(t, x)=n \int \frac{\partial f}{\partial t}(t, x-y) g(n y) d y .
$$

As $n \rightarrow \infty, \lim F_{n}(t, x)=F(t, x)$,

$$
\begin{gathered}
\lim \frac{\partial F_{n}}{\partial t}(t, x)=\frac{\partial F}{\partial t}(t, x) \\
\lim \frac{\partial F_{n}}{\partial x}(t, x)=f(t, x) \quad \text { a.e. }
\end{gathered}
$$

and

$$
\lim \frac{\partial^{2} F_{n}}{\partial t \partial x}(t, x)=\frac{\partial f}{\partial t}(t, x) \quad \text { a.e. }
$$

Applying Theorem 6 to $F_{n}(t, x)$

$$
\begin{aligned}
F_{n}\left(t, X_{t}\right)= & F_{n}\left(0, X_{0}\right)+\int_{0}^{t} \frac{\partial F_{n}}{\partial t}\left(s, X_{s}\right) d s+\int_{0}^{t} \frac{\partial F_{n}}{\partial x}\left(s, X_{s}\right) d X_{s} \\
& -\frac{1}{2} \int_{-\infty}^{\infty} \frac{\partial F_{n}}{\partial x}(t, a) d_{a} L_{t}^{a}+\frac{1}{2} \int_{0}^{t}\left(\int_{-\infty}^{\infty} \frac{\partial^{2} F_{n}}{\partial t d x}(s, a) d_{a} L_{s}^{a}\right) d s .
\end{aligned}
$$

Letting $n \rightarrow \infty$ we have

$$
\begin{aligned}
F\left(t, X_{t}\right)= & F\left(0, X_{0}\right)+\int_{0}^{t} \frac{\partial F}{\partial t}\left(s, X_{s}\right) d s+\int_{0}^{t} f\left(s, X_{s}\right)_{s} d X_{s} \\
& -\frac{1}{2} \int_{-\infty}^{\infty} f(t, a) d_{a} L_{t}^{a}+\frac{1}{2} \int_{0}^{t}\left(\int_{-\infty}^{\infty} \frac{\partial f}{\partial t}(s, a) d_{a} L_{s}^{a}\right) d s .
\end{aligned}
$$

Remarks 9. This corollary holds without the hypothesis that $F(t, 0)$ $=0$; suppose $F(t, x)$ satisfies the hypotheses of the corollary except possibly the condition $F(t, 0)=0$. Then $G(t, x)=F(t, x)-F(t, 0)$ satisfies all the hypotheses, and so the result holds for $G$. However,

$$
\frac{\partial G}{\partial t}(t, x)=\frac{\partial F}{\partial t}(t, x)-\frac{\partial F}{\partial t}(t, 0)
$$

and the integral in $s$ then contributes an additional quantity

$$
\int_{0}^{t}-\frac{\partial F}{\partial t}(s, 0) d s=F(0,0)-F(t, 0)
$$

so cancelling the extra terms.

The next result extends some formulae of Yamada [4], and Proposition 3.1 of Yor [5]. First we give a definition. 
Suppose $B_{t}, t \geq 0$ is a standard Brownian motion and $F(t, x)$ is such that it is $C^{1}$ in $t$ and $\partial F / \partial x$ exists and belongs to $L_{\mathrm{loc}}^{2}([0, \infty) \times R)$. Then the second derivative $\partial^{2} F / \partial x^{2}$ exists in the sense of distribution theory.

Definition 10. The process

$$
A_{t}^{F}=\int_{0}^{t} \frac{\partial^{2} F}{\partial x^{2}}\left(s, B_{s}\right) d s
$$

is defined to be

$$
2\left(F\left(t, B_{t}\right)-F(0,0)-\int_{0}^{t} \frac{\partial F}{\partial x}\left(s, B_{s}\right) d B_{s}-\int_{0}^{t} \frac{\partial F}{\partial t}\left(s, B_{s}\right) d s\right) .
$$

Theorem 11. Suppose for $(t, x) \in[0, \infty) \times R F(t, x)$ is continously differentiable in $t$ and twice continuously differentiable in $x$ outside the origin.

Write $(\partial F / \partial x)(t, x)=f(t, x)$ and, for some $T>0$, suppose that

$$
f^{*}(x)=\sup _{t \leq T}|f(t, x)| \in L_{\mathrm{loc}}^{2}(R)
$$

and

$$
\frac{\partial f^{*}}{\partial t}(x)=\sup _{t \leq T}\left|\frac{\partial f}{\partial t}(t, x)\right| \in L_{10 \mathrm{c}}^{1}(R) .
$$

Then for all $p \in[1, \infty)$

$$
\begin{aligned}
\lim _{\varepsilon \rightarrow 0} E\left[\sup _{t \leq T} \mid A_{t}^{F}\right. & -\left\{\int_{0}^{t} \frac{\partial^{2} F}{\partial x^{2}}\left(s, B_{s}\right) I_{\left|B_{s}\right| \geq \varepsilon} d s\right. \\
& \left.\left.\left.+\int_{0}^{t} f(s, \varepsilon) d_{s} L_{s}^{\varepsilon}-\int_{0}^{t} f(s,-\varepsilon) d_{s} L_{s}^{-\varepsilon}\right\}\right\}^{p}\right]=0 .
\end{aligned}
$$

Proof. Without loss of generality suppose that $F(t, 0)=0$ so

$$
F(t, x)=\int_{0}^{x} f(t, y) d y
$$

and

$$
\frac{\partial F}{\partial t}(t, x)=\int_{0}^{x} \frac{\partial f}{\partial t}(t, y) d y .
$$

Write $f_{\varepsilon}(t, y)=f(t, y) I_{|y| \geq \varepsilon}$ and

$$
F_{\varepsilon}(t, x)=\int_{0}^{x} f_{\varepsilon}(t, y) d y .
$$


Then

$$
\frac{\partial F_{\varepsilon}}{\partial t}(t, x)=\int_{0}^{x} \frac{\partial f_{\varepsilon}}{\partial t}(t, y) d y
$$

and applying Corollary 8 to $F_{\varepsilon}$ with $X$ a standard Brownian motion $B$

$$
\begin{aligned}
F_{\varepsilon}\left(t, B_{t}\right)= & \int_{0}^{t} f_{\varepsilon}\left(s, B_{s}\right) d B_{s}+\int_{0}^{t} \frac{\partial F_{\varepsilon}}{\partial t}\left(s, B_{s}\right) d s \\
& -\frac{1}{2} \int_{-\infty}^{\infty} f_{\varepsilon}(t, a) d_{a} L_{t}^{a}+\frac{1}{2} \int_{0}^{t} \int_{-\infty}^{\infty} \frac{\partial f_{\varepsilon}}{\partial t}(s, a) d_{a} L_{s}^{a} d s
\end{aligned}
$$

Writing

$$
A_{t}^{F_{\varepsilon}}=-\int_{-\infty}^{\infty} f_{\varepsilon}(t, a) d_{a} L_{t}^{a}+\int_{0}^{t} \int_{-\infty}^{\infty} \frac{\partial f_{\varepsilon}}{\partial s}(s, a) d_{a} L_{s}^{a} d s
$$

we have

$$
A_{t}^{F_{\varepsilon}}=-\left(\int_{\varepsilon}^{\infty}+\int_{-\infty}^{-\varepsilon} f(t, a) d_{a} L_{t}^{a}\right)+\int_{0}^{t}\left(\int_{\varepsilon}^{\infty}+\int_{-\infty}^{-\varepsilon} \frac{\partial f}{\partial t}(s, a) d_{a} L_{s}^{a}\right) d s,
$$

and by parts (in $a$ ) this is

$$
\begin{aligned}
= & L_{t}^{\varepsilon} f(t, \varepsilon)-L_{t}^{-\varepsilon} f(t,-\varepsilon)+\left(\int_{\varepsilon}^{\infty}+\int_{-\infty}^{-\varepsilon} \frac{\partial^{2} F}{\partial x^{2}}(t, a) L_{t}^{a} d a\right) \\
& -\int_{0}^{t}\left(L_{s}^{\varepsilon} \frac{\partial f}{\partial t}(s, \varepsilon)-L_{s}^{-\varepsilon} \frac{\partial f}{\partial s}(s,-\varepsilon)\right) d s \\
& -\int_{0}^{t}\left(\int_{\varepsilon}^{\infty}+\int_{-\infty}^{-\varepsilon} \frac{\partial^{2} f}{\partial t \partial x}(s, a) L_{s}^{a} d a\right) d s .
\end{aligned}
$$

Applying Fubini's Theorem to the final term and integrating by parts in $s$

$$
\begin{aligned}
\left(\int_{\varepsilon}^{\infty}+\right. & \left.\int_{-\infty}^{-\varepsilon}\right)\left(\int_{0}^{t} \frac{\partial^{2} f}{\partial t d x}(s, a) L_{s}^{a} d s\right) d a \\
& =\left(\int_{\varepsilon}^{\infty}+\int_{-\infty}^{-\varepsilon}\right)\left(L_{t}^{a} \frac{\partial^{2} F}{\partial x^{2}}(t, a)-\int_{0}^{t} \frac{\partial^{2} F}{\partial x^{2}}(s, a) d_{s} L_{s}^{a}\right) d a
\end{aligned}
$$

Therefore,

$$
\begin{aligned}
A_{t}^{F_{\varepsilon}}= & -\int_{-\infty}^{\infty} f_{\varepsilon}(t, a) d_{a} L_{t}^{a}+\int_{0}^{t}\left(\int_{-\infty}^{\infty} \frac{\partial f_{\varepsilon}}{\partial t}(s, a) d_{a} L_{s}^{a}\right) d s \\
= & L_{t}^{\varepsilon} f(t, \varepsilon)-L_{t}^{-\varepsilon} f(t,-\varepsilon)-\int_{0}^{t} L_{s}^{\varepsilon} \frac{\partial f}{\partial t}(s, \varepsilon) d s \\
& +\int_{0}^{t} L_{s}^{-\varepsilon} \frac{\partial f}{\partial t}(s,-\varepsilon) d s+\int_{0}^{t} \frac{\partial^{2} F}{\partial x^{2}}\left(s, B_{s}\right) I_{\left|B_{s}\right| \geq \varepsilon} d s \\
= & \int_{0}^{t} f(s, \varepsilon) d_{s} L_{s}^{\varepsilon}-\int_{0}^{t} f(s,-\varepsilon) d_{s} L_{s}^{-\varepsilon}+\int_{0}^{t} \frac{\partial^{2} F}{\partial x^{2}}\left(s, B_{s}\right) I_{\left|B_{s}\right| \geq \varepsilon} d s
\end{aligned}
$$


For the function $F(t, x)$ the process $A_{t}^{F}$ is defined by

$$
A_{t}^{F}=2\left(F\left(t, B_{t}\right)-\int_{0}^{t} f\left(s, B_{s}\right) d B_{s}-\int_{0}^{t} \frac{\partial F}{\partial t}\left(s, B_{s}\right) d s\right)
$$

Therefore,

$$
\begin{aligned}
A_{t}^{F}-A_{t}^{F_{\varepsilon}}= & 2\left(\int_{0}^{B_{t}} f(t, y) I_{|y| \leq \varepsilon} d y-\int_{0}^{t} f\left(s, B_{s}\right) I_{\left|B_{s}\right| \leq \varepsilon} d B_{s}\right. \\
& \left.-\int_{0}^{t} \int_{0}^{B_{s}} \frac{\partial f}{\partial t}(s, y) I_{|y| \leq \varepsilon} d y d s\right),
\end{aligned}
$$

and for $p \in[1, \infty), T>0$,

$$
\begin{aligned}
E\left[\sup _{t \leq T}\left|A_{t}^{F}-A_{t}^{F_{s}}\right|^{p}\right] \leq & \text { Const } E\left[\sup _{t \leq T}\left|\int_{0}^{B_{t}} f(t, y) I_{|y| \leq \varepsilon} d y\right|^{p}\right. \\
& +\sup _{t \leq T}\left|\int_{0}^{t} f\left(s, B_{s}\right) I_{\left|B_{s}\right| \leq \varepsilon} d B_{s}\right|^{p} \\
& \left.+\sup _{t \leq T}\left|\int_{0}^{t}\left(\int_{0}^{B_{s}} \frac{\partial f}{\partial t}(s, y) I_{|y| \leq \varepsilon} d y\right) d s\right|^{p}\right] .
\end{aligned}
$$

Denote the three terms in the expectation by $I^{(1)}, I^{(2)}$ and $I^{(3)}$, respectively.

Then

$$
E\left[I^{(1)}\right] \leq E\left[\sup _{t \leq T}\left(\int_{-\varepsilon}^{e}|f(t, y)| d y\right)^{p}\right] \leq\left(\int_{-\varepsilon}^{\varepsilon} f^{*}(y) d y\right)^{p},
$$

and this converges to 0 as $\varepsilon \rightarrow 0$.

$$
\begin{aligned}
E\left[I^{(2)}\right] & \leq C_{p} E\left[\left|\int_{0}^{T} f\left(s, B_{s}\right) I_{\left|B_{s}\right| \leq \varepsilon} d B_{s}\right|^{p}\right] \leq \text { Const } E\left(\int_{0}^{T} f^{2}\left(s, B_{s}\right) I_{\left|B_{s}\right| \leq \varepsilon} d s\right)^{p / 2} \\
& =\text { Const } E\left(\int_{-\infty}^{\infty}\left(\int_{0}^{T} f^{2}(s, a) I_{|a| \leq \varepsilon} d_{s} L_{s}^{a}\right) d a\right)^{p / 2} \\
& \leq \text { Const } E\left(\int_{-\varepsilon}^{\varepsilon} f^{*}(a)^{2} L_{T}^{a} d a\right)^{p / 2} \leq \operatorname{Const}\left(E\left(L_{T}^{*}\right)^{p / 2}\right)\left(\int_{-\varepsilon}^{\varepsilon} f^{*}(a)^{2} d a\right)^{p / 2},
\end{aligned}
$$

which again converges to 0 as $\varepsilon \rightarrow 0$.

Finally,

$$
E\left[I^{(3)}\right] \leq E\left[\sup _{t \leq T}\left|\int_{0}^{t} \int_{-\varepsilon}^{\varepsilon}\right| \frac{\partial f}{\partial t}(s, y)|d y d s|^{p}\right] \leq T^{p}\left(\int_{-\varepsilon}^{\varepsilon} \frac{\partial f^{*}}{\partial t}(y) d y\right)^{p}
$$

which converges to 0 as $\varepsilon \rightarrow 0$, so the result is proved.

ExAmples 12. Suppose $B_{t}, t \geq 0$, is a standard Brownian motion. 
1) Taking $F\left(t, B_{t}\right)=\exp \left(\lambda B_{t}-\lambda^{2} t / 2\right)$, for $\lambda \in R$, from the identity obtained in Theorem 6

$$
\begin{aligned}
& \int_{0}^{t} \lambda \exp \left(\lambda B_{s}-\lambda^{2} s / 2\right) d s \\
& \quad=-e^{-\lambda 2 t / 2} \int_{-\infty}^{\infty} e^{\lambda a} d_{a} L_{t}^{a}-\lambda^{2} / 2 \int_{0}^{t} e^{-\lambda^{2} s / 2}\left(\int_{-\infty}^{\infty} e^{\lambda a} d_{a} L_{s}^{a} d s\right) .
\end{aligned}
$$

2) With $F(t, x)=\left\{\begin{array}{cc}\phi(t)(x \log x-x) & \text { for } x>0 \\ 0 & \text { for } x \leq 0\end{array}\right.$

where $\phi$ is $C^{1}$ in $t$

$$
\frac{\partial^{2} F}{\partial^{2} x}(t, x)=\phi(t) / x \quad \text { for } x>0
$$

and Theorem 11 implies that in $L^{p}, p \in[1, \infty)$,

$$
\begin{aligned}
A_{t}^{F} & =\text { Principal value of } \int_{0}^{t} \frac{\phi(s)}{\left(B_{s}\right)_{+}} d s \\
& =\lim _{\varepsilon \rightarrow 0}\left\{\int_{0}^{t} \frac{\phi(s)}{B_{s}} I_{B_{s} \geq \varepsilon} d s+\log \varepsilon \int_{0}^{t} \phi(s) d_{s} L_{s}^{\varepsilon}\right\} .
\end{aligned}
$$

3) With $F(t, x)=\left\{\begin{array}{cc}\phi(t)|x|^{\lambda+2} /(\lambda+1)(\lambda+2) & \text { for } x>0 \\ 0 & \text { for } x \leq 0\end{array}\right.$

where $-3 / 2<\lambda<-1$ and $\phi$ is $C^{1}$ in $t$, we have from Theorem 11 that in $L^{p}, p \in[1, \infty)$,

$$
\begin{aligned}
A_{t}^{F} & =\text { Finite part of } \int_{0}^{t} \phi(s)\left|B_{s}\right|^{\lambda} d s \\
& =\lim _{\varepsilon \rightarrow 0}\left\{\int_{0}^{t} \phi(s)\left|B_{s}\right|^{\mid} I_{\left|B_{s}\right| \geq \varepsilon} d s+\frac{\varepsilon^{\lambda+1}}{(\lambda+1)} \int_{0}^{t} \phi(s) d_{s} L_{s}^{\varepsilon}-\frac{\varepsilon^{\lambda+1}}{(\lambda+1)} \int_{0}^{t} \phi(s) d_{s} L_{s}^{-\varepsilon}\right\} .
\end{aligned}
$$

Acknowledgement. The authors are grateful to Dr. M. Yor for his comments on an earlier version of this paper.

\section{REFERENCES}

[1] Bouleau, N. and Yor, M., Sur la variation quadratique des temps locaux de certaines semimartingales, C.R. Acad. Sci. Paris, 292 (1981), 491-494.

[2] Meyer, P. A., Un cours sur les integrales stochastiques, Sem de Probabilités X, Lec. Notes in Math., 511, 245-400.

[ 3 ] Perkins, E., Local time is a semimartingale, Z. Wahrsch. Verw. Gebiete, 60 (1982), 79-117. 
[4] Yamada, T., On some representations concerning stochastic integrals, to appear.

[5] Yor, M., Sur la transformation de Hilbert des temps locaux Browniens, et une extension de la Formule d'Ito, Sem de Probabilités XVI, Lec. Notes in Math., 920, 238-247.

Department of Statistics and Applied Probability University of Alberta, Edmonton, Canada T6G 2 G1 